\title{
ОПТИМАЛЬНЫЙ СИНТЕЗ ПРИВОДНЫХ ШАРНИРНЫХ СОЕДИНЕНИЙ ГИДРАВЛИЧЕСКИХ МАНИПУЛЯЦИОННЫХ СИСТЕМ МОБИЛЬНЫХ КРАНОВ
}

\author{
А.В.ЛАГЕРЕВ, И.А.ЛАГЕРЕВ
}

Брянский государственный университет имени академика И.Г.Петровского, Брянск, Россия

Рассмотрены конструкция, особенности работы и методика оптимального проектирования приводного шарнирного соединения, предложенного в качестве альтернативного конструктивного варианта шарнирного соединения смежных звеньев гидравлических манипуляционных систем мобильных стреловых крановманипуляторов. Конструкция устройства позволяет совместить две функции: обеспечить постоянную неразрывную кинематическую связь между смежными звеньями манипуляционной системы путем создания цилиндрического шарнира для выполнения возвратно-поворотного относительного движения смежных звеньев и сформировать встроенный поворотный гидродвигатель шиберного типа. Разработана математическая модель, сформулирована и решена задача оптимального синтеза приводного шарнирного соединения на основе минимизации массы устройства при соблюдении нелинейной системы конструкторских, монтажных, силовых, прочностных и кинематических ограничений. Анализ результатов выполненных оптимизационных расчетов показал, что масса оптимального варианта устройства возрастает с ростом преодолеваемого момента от перемещаемого груза и снижается с увеличением числа камер шарнирного соединения. Рабочее давление гидросистемы не оказывает практического влияния на оптимальную массу, что позволяет использовать для обеспечения движения звеньев манипуляционной системы насосный агрегат меньшей мощности. Оптимальные значения основных конструктивных размеров приводного шарнирного соединения определяются как величиной эксплуатационной нагрузки, так и условиями монтажа устройства с учетом размеров поперечных сечений смежных звеньев манипуляционной системы. При проектировании манипуляционных систем приводные шарнирные соединения позволяют отказаться от выносных силовых гидродвигателей, исключить эксплуатационные отказы вследствие изнашивания и усталостного разрушения элементов шарниров, а также повысить энергоэффективность кранов-манипуляторов путем перевода гидросистемы на более низкие уровни рабочего давления при сохранении требуемых грузо-высотных характеристик.

Ключевые слова: манипуляционная система; мобильный кран; приводное шарнирное соединение; гидравлический привод; оптимизация; синтез; масса

Как цитировать эту статью: Лагерев А.В. Оптимальный синтез приводных шарнирных соединений гидравлических манипуляционных систем мобильных кранов / А.В.Лагерев, И.А.Лагерев // Записки Горного института. 2018. Т. 232. С. 413-420. DOI: 10.31897/PMI.2018.4.413

Введение. Гидравлические манипуляционные системы (стреловые краны-манипуляторы), установленные на мобильных транспортно-технологических машинах различного назначения, получили широкое распространение во многих отраслях экономики - промышленном производстве, горной промышленности, газо- и нефтедобыче, строительстве и др. [1]. В России они являются весьма востребованным видом грузоподъемного оборудования [7]. На мировом рынке представлены преимущественно конструкции таких стран-производителей, как Южная Корея, Япония, Китай, Германия, Италия, США, Австрия, Россия и др. [2].

Несущая металлоконструкция манипуляционной системы стрелового крана-манипулятора состоит из 3-12 последовательно расположенных подвижных звеньев, соединенных попарно цилиндрическими или призматическими шарнирами [3]. Конструктивно цилиндрические шарниры представляют собой закрепленные на концах смежных звеньев проушины, имеющие соосные отверстия для установки шарнирного пальца $[7,20]$. Такая конструкция обеспечивает лишь неразрывность кинематической цепи манипуляционной системы. Для совершения звеньями относительного движения необходима установка силовых гидроцилиндров, крепящихся при помощи попарно расположенных проушин к поверхности смежных звеньев [22].

Постановка проблемы. По мере эксплуатации цилиндрических шарнирных соединений происходит увеличение зазоров между шарнирными пальцами и поверхностями отверстий проушин вследствие изнашивания и смятия их контактирующих поверхностей $[7,11,12]$. Это приводит к повышению уровня напряжений в металлоконструкции крана до двух и более раз и линейного ускорения перемещения груза до 20 раз $[16,17]$. Использование промежуточных антифрикционных втулок [3], полимерных покрытий $[8,14]$ или различных методов упрочнения [15] способно замедлить, но не исключить развитие негативных процессов [11]. Наличие выносных гидродвигателей механизмов движения снижает грузоподъемность [7] и объем рабочей зоны крана-манипулятора [3]. Элементы узлов крепления силовых гидродвигателей к звеньям подвержены отказам вследствие возникновения в них усталостных трещин [7, 12]. 


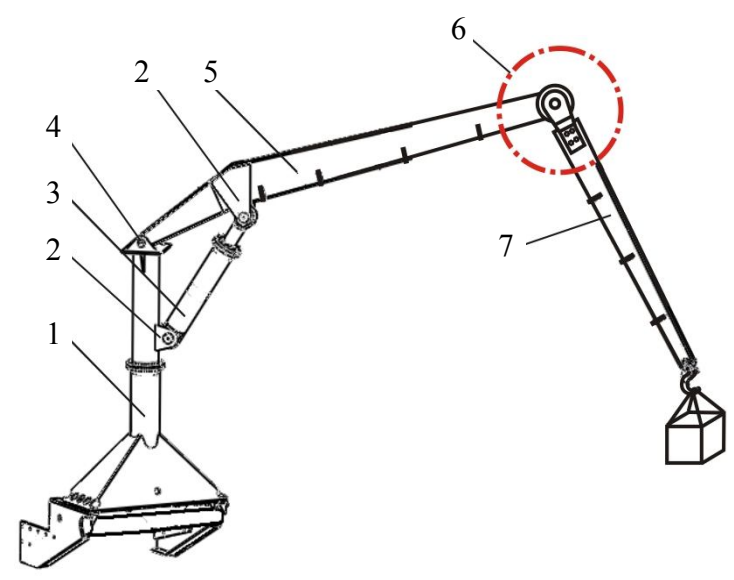

Рис.1. Трехзвенная манипуляционная система мобильного стрелового крана-манипулятора с приводным шарнирным соединением

Альтернативным вариантом по отношению к традиционной конструкции шарнирных соединений звеньев манипуляционных систем мобильных крановманипуляторов может выступать конструкция приводных шарнирных соединений [10].

Конструкция и принцип работы приводных шарнирных соединений. Внешний вид манипуляционной системы стрелового крана-манипулятора с традиционным и приводным шарнирными соединениями смежных звеньев показан на рис.1. Шарнирное соединение 4 колонны 1 и стрелы 5 имеет традиционную конструкцию с установкой силового гидроцилиндра 3 с помощью элементов крепления 2 к смежным звеньям. Приводное шарнирное соединение 6 стрелы 5 и рукояти 7 обеспечивает поворотное движение рукояти относительно стрелы, не требуя установки выносного гидроцилиндра.

На рис.2 показана конструкция приводного шарнирного соединения [10], на рис.3 - его расчетная схема. Конструктивно приводное шарнирное соединение состоит из корпуса 5, с обоих торцов герметично закрываемого съемными крышками 13. Корпус неподвижно закрепляется на конце одного из смежных звеньев манипуляционной системы. Внутри корпуса в опорных подшипниках качения 12 располагается ротор 4. С его обеих сторон нарезаются эвольвентные шлицы для соединения ротора с хомутами 10. Другие концы хомутов с помощью шпилек 9 закрепляются на боковых поверхностях второго смежного звена. Внутри корпуса неподвижно устанавливается три, четыре или пять перегородок 11 на равном угловом расстоянии $\alpha$. На таком же расстоянии $\alpha$ на поверхности втулки 3 закрепляется равное количество лопастей 2 . Например, в результате установки трех перегородок образуется шесть камер: $U_{1}, U_{2}, U_{3}$ и $V_{1}, V_{2}, V_{3}$. Каждая из них подключается индивидуальным трубопроводом к гидравлической системе кранаманипулятора, причем трубопроводы камер $U_{1}, U_{2}, U_{3}$ являются подъемными 6 , а камер $V_{1}, V_{2}$, $V_{3}$ - опускными трубопроводами 7. Герметизация стыковочных поверхностей деталей достигается комплексным подходом на основе как современных уплотнительных устройств, так и повышения точности их механической обработки с помощью виброустойчивых инструментальных систем $[9,18]$.

Таким образом, функцию шарнирного соединения смежных звеньев реализуют корпус, торцевые крышки, ротор и хомуты. Контактирование этих элементов обеспечивает постоянную
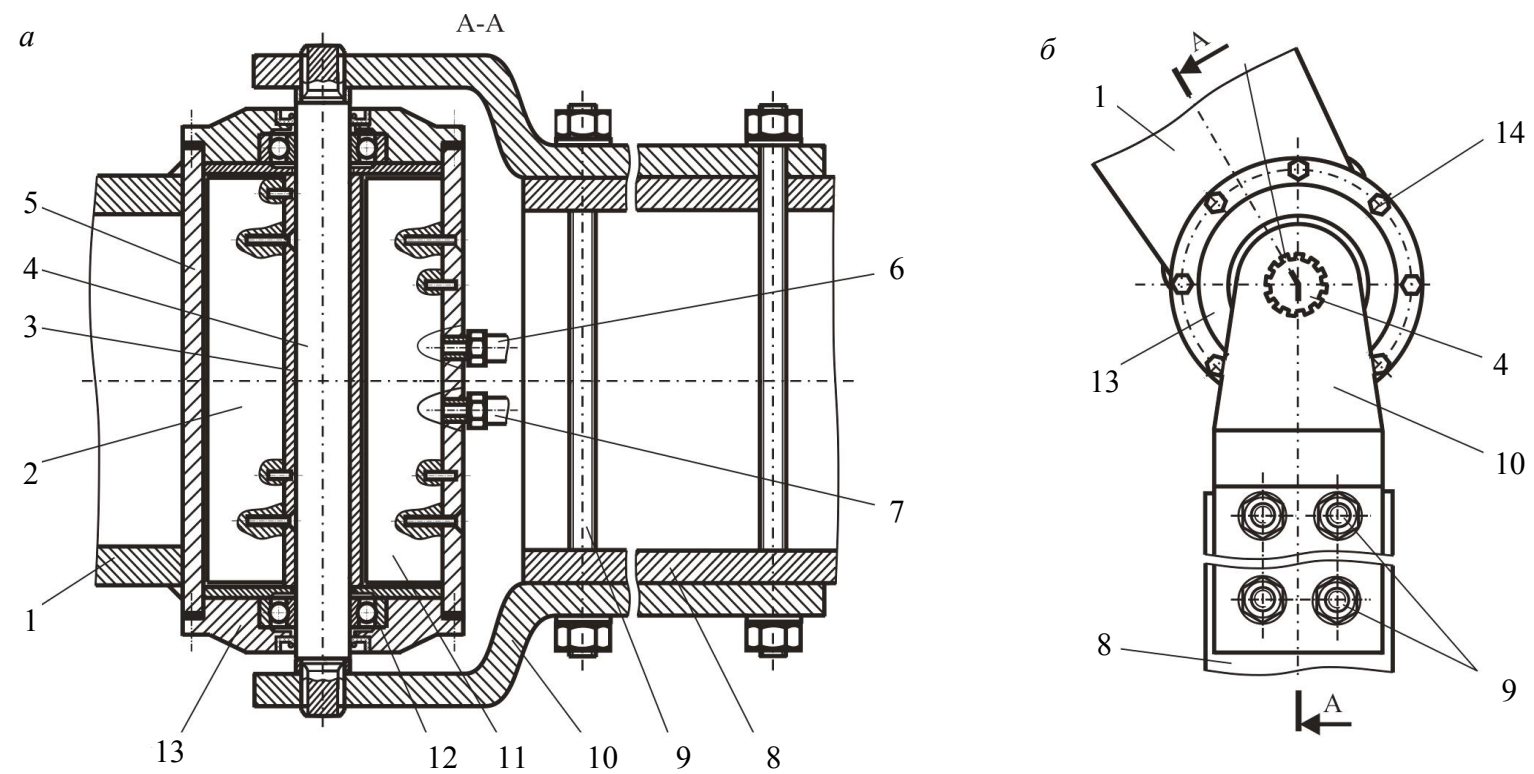

Рис.2. Конструкция приводного шарнирного соединения: $a$ - продольный разрез; $\sigma$ - вид сбоку 
неразрывную кинематическую связь между смежными звеньями и формирует цилиндрический шарнир. Функцию силового гидроцилиндра реализуют корпус с закрепленными на нем подъемными и опускными трубопроводами, торцевые крышки, перегородки, лопасти, ротор и уплотнительные элементы. Они формируют встроенный гидродвигатель, аналогичный по принципу действия шиберным поворотным гидродвигателям [4, 13, 21].

При подключении подъемных трубопроводов к напорной гидролинии гидросистемы крана-манипулятора рабочая жидкость под высоким давлением $p_{1}$ поступает в камеры $U_{1}, U_{2}$, $U_{3}$. Одновременно с этим опускные трубопроводы камер $V_{1}, V_{2}, V_{3}$ подключаются к сливной гидролинии под низким давлением $p_{2}$. В результате разности давления $\Delta p=p_{1}-p_{2}$ рабочей жидкости, находящейся в смежных камерах $U_{1}-V_{1}, U_{1}-V_{1}, U_{2}-V_{2}, U_{3}-V_{3}$, появляются силы $R_{1}, R_{2}, R_{3}$. Они создают относительно продольной оси ротора (точки O) суммарный крутящий момент

$$
T_{t}=R_{1} h+R_{2} h+R_{3} h=n_{h} \Delta p A h,
$$

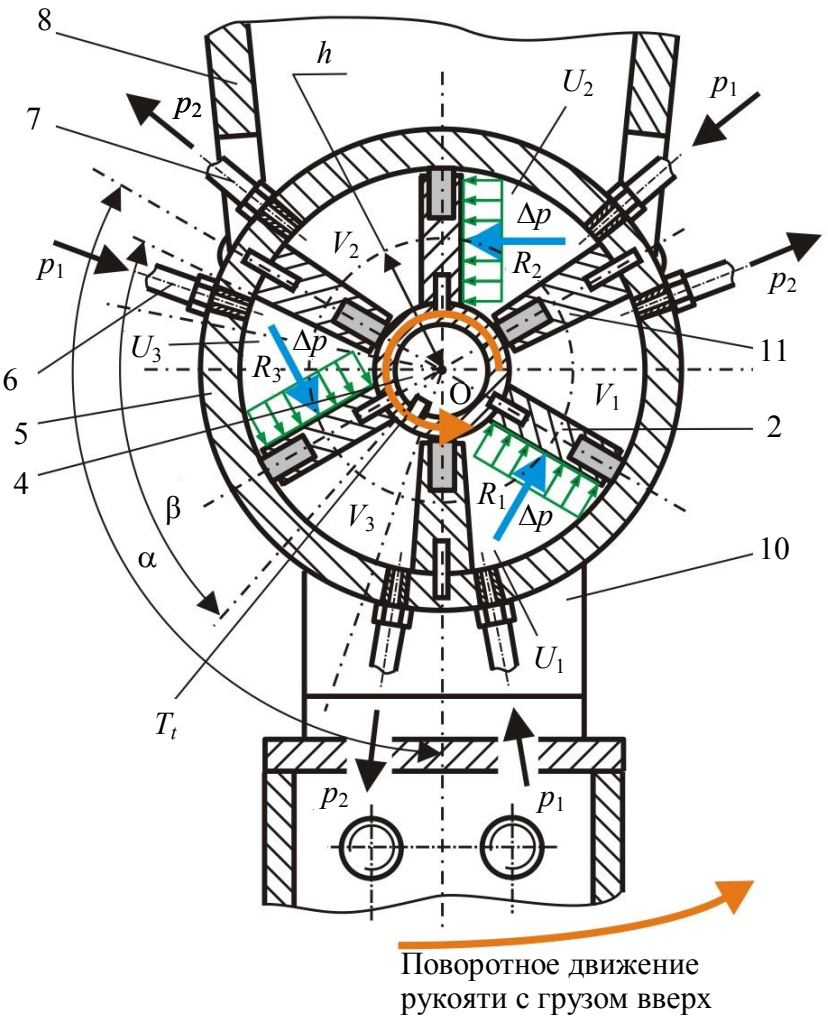

Рис.3. Расчетная схема шестикамерного приводного шарнирного соединения

где $A$ - площадь боковой поверхности лопасти; $h$ - плечо действия равнодействующей силы $R_{i}$ относительно продольной оси ротора; $n_{h}$ - число лопастей.

Максимальный угол поворота рукояти определяется наибольшим возможным углом поворота лопасти между двумя соседними перегородками и ориентировочно составляет $\beta \approx(0,70-85) \alpha$.

Постановка задачи оптимального синтеза приводных шарнирных соединений. Масса элементов приводного шарнирного соединения повышает массу подвижных конструктивных элементов манипуляционной системы. Поэтому при проектировании приводного шарнирного соединения важно обеспечить минимальный вклад его элементов в общую массу металлоконструкции шарнирно-сочлененной манипуляционной системы крана.

Масса приводного шарнирного соединения складывается из масс корпуса, торцевых крышек, перегородок, лопастей, ротора, втулки, подшипников качения, соединительных шпилек и заполняющей камеры рабочей жидкости:

$$
\begin{gathered}
M \approx \pi \rho_{b}\left(D+s_{w}\right) s_{w} H+0,5 \pi \rho_{l}\left[\left(D+2 s_{w}\right)^{2}-d_{s}^{2}\right] s_{l}+0,5 \rho_{v}\left[0,5 \pi\left(d_{v}-d_{s}\right)^{2}+n_{h}\left(D-d_{v}\right) h_{v} H\right]+ \\
+0,25 \pi \rho_{s} d_{s}^{2}\left[H+2\left(s_{l}+s_{b t}\right)\right]+2 M_{b r, 1}\left(d_{s}\right)+0,5 n_{h} \rho_{d}\left(D-d_{v}\right) h_{d} H+\rho_{b t} \frac{n_{T} T_{t}\left[H+2\left(s_{b t}+d_{b t}\right)\right]}{2[\tau]_{s} h_{b t}}+ \\
+0,5 \rho_{h f}\left(D-d_{v}\right)\left[0,5 \pi\left(D+d_{v}\right)-n_{h}\left(h_{d}+h_{v}\right)\right] H,
\end{gathered}
$$

где $\rho_{b}, \rho_{l}, \rho_{d}, \rho_{v}, \rho_{s}, \rho_{b t}, \rho_{h f}$ - плотность материала корпуса, торцевых крышек, перегородок, лопастей, втулки, ротора, шпилек и рабочей жидкости; $D, H, s_{w}$ - внутренний диаметр, длина и толщина стенки корпуса; $s_{l}$ - толщина торцевой крышки; $d_{v}, d_{s}, d_{b t}-$ диаметры втулки, ротора и шпильки; $h_{d}, h_{v}$ - толщина перегородки и лопасти; $s_{b t}$ - хомута; $h_{b t}$ - расстояние от осевой линии сечения конечного звена до центра сечения шпильки; $n_{T}$ - коэффициент запаса по преодолеваемому крутящему моменту $\left(n_{T}>1\right) ; M_{b r, 1}\left(d_{s}\right)$ - масса подшипника, определяемая его типоразмером. 
Согласно зависимости (1) масса приводного шарнирного соединения в условиях действия эксплуатационной нагрузки (крутящего момента $T_{t}$ и рабочего давления $p_{1}$ ) зависит от 11 конструктивных параметров: $D, H, s_{w}, s_{l}, s_{b t}, d_{v}, d_{s}, d_{b t}, h_{d}, h_{v}, n_{h}$. Учитывая рекомендации [6], два из перечисленных параметров целесообразно рассматривать как параметры независимые и определяющие размеры всего устройства - внутренний диаметр $D$ и длина корпуса $H$. В качестве независимого параметра также целесообразно рассматривать диаметр ротора $d_{s}$, так как он позволяет определить конструктивные размеры соединительных элементов устройства с концевым звеном. Остальные размеры либо являются фиксированными, так как определяются конструкцией приводного шарнирного соединения и величиной эксплуатационной нагрузки $\left(s_{b t}, d_{b t}, n_{h}\right)$, либо однозначно определяются в зависимости от $D, H$ и $d_{s}\left(s_{w}, s_{l}, d_{v}, h_{d}, h_{v}\right)$. Число лопастей шибера $n_{h}$ удобно задавать в качестве исходного данного при каждом оптимизационном расчете, задавая последовательно его возможные значения из полного множества значений $n_{h}=3,4,5$.

Таким образом, при формулировании задачи оптимального синтеза приводного шарнирного соединения в качестве вектора переменных (варьируемых) параметров оптимизации следует использовать внутренний диаметр, длину корпуса и диаметр ротора, сформировав их них вектор неизвестных

$$
\{x\}^{T}=\left\{x_{1} x_{2} x_{3}\right\}=\left\{D H d_{s}\right\} .
$$

Остальные параметры являются неуправляемыми (фиксированными), из которых формируется вектор

$$
\{z\}^{T}=\left\{z_{1} z_{2} z_{3} z_{4} z_{5} z_{6} z_{7}\right\}=\{z\}^{T}=\left\{z_{1} z_{2} z_{3} z_{4} z_{5} z_{6} z_{7}\right\} .
$$

Окончательно задача оптимизации гидравлического приводного шарнирного соединения смежных звеньев манипуляционной системы сводится к минимизации целевой функции вида

$$
\begin{gathered}
F(\{x\},\{z\})=\pi \rho_{b}\left(x_{1}+z_{1}\right) z_{1} x_{2}+0,5 \pi \rho_{l}\left[\left(x_{1}+2 z_{1}\right)^{2}-x_{3}^{2}\right] z_{2}+0,5 n_{h} \rho_{d}\left(x_{1}-z_{4}\right) z_{5} x_{2}+ \\
+\rho_{b t} \frac{n_{T} T_{t}\left[x_{2}+2\left(z_{3}+z_{7}\right)\right]}{2[\tau]_{s} h_{b t}}+0,5 \rho_{v}\left[0,5 \pi\left(z_{4}-x_{3}\right)^{2}+n_{h}\left(x_{1}-z_{4}\right) z_{6} x_{2}\right]+ \\
+0,25 \pi \rho_{s} x_{3}^{2}\left[x_{2}+2\left(z_{2}+z_{3}\right)\right]+2 M_{b r, 1}\left(x_{3}\right)+0,5 \rho_{h f}\left(x_{1}-z_{4}\right)\left[0,5 \pi\left(x_{1}+z_{4}\right)-n_{h}\left(z_{5}+z_{6}\right)\right] x_{2}
\end{gathered}
$$

при следующих ограничениях:

• конструктивными условиями

$$
\begin{gathered}
x_{1}>0 ; \quad x_{2}>0 ; \quad x_{3}>0 ; \\
\left(x_{1}+2 z_{1}\right)-0,8 H_{A} \geq 0 ; \quad 1,5 H_{A}-\left(x_{1}+2 z_{1}\right) \geq 0 ; \\
1,3 B_{A}-x_{2} \geq 0 ; \quad 1,5 B_{A}-\left(x_{2}+2 z_{2}\right) \geq 0 ; \quad x_{1}-z_{4} \geq 0 ; \\
\left(x_{2}+2 z_{2}\right)-B_{A} \geq 0 ; \\
z_{3}-0,8 s_{B} \geq 0 ; \\
2 s_{B}-z_{3} \geq 0 ; \\
z_{1}-s_{A} \geq 0 ;
\end{gathered}
$$

- условием недопущения смятия рабочих поверхностей шпонок соединения ротора со втулкой

$$
[\sigma]_{b r, k}-3,33 \frac{n_{T} T_{t}}{x_{3}^{2}\left(z_{4}-x_{3}\right)} \geq 0
$$

• условием обеспечения максимального угла относительного поворота конечного звена

$$
\frac{2 \pi}{n_{h}}-\frac{\left(z_{5}+z_{6}\right) n_{h}}{x_{1}}-[\varphi] \geq 0 ;
$$

- условием обеспечения тягового усилия 


$$
0,125 \Delta p n_{h} x_{2}\left(x_{1}^{2}-z_{4}^{2}\right)-n_{T} T_{t} \geq 0 ;
$$

- условием лимитирования углового ускорения ротора при разгоне

$$
0,125 \Delta p n_{h} x_{2}\left(x_{1}^{2}-z_{4}^{2}\right)-T_{t}-J_{\text {и }}[\ddot{\varphi}] \geq 0 ;
$$

- условием обеспечения прочности на кручение шлицевого участка ротора

$$
x_{3}-2 \sqrt[3]{\frac{2 K_{\tau s} n_{T} T_{t}}{\pi[\tau]_{t}}} \geq 0 ;
$$

• условием обеспечения прочности на смятие рабочей поверхности эвольвентных шлицев ротора

$$
x_{3}-3,16 \sqrt{\frac{n_{T} T_{t}}{\psi z_{3} \sigma_{u s}}}-m_{g} \geq 0
$$

- условием обеспечения усталостной прочности корневого сечения лопасти

$$
\frac{\sigma_{-1}}{K}-\frac{3 \Delta p\left(x_{1}-z_{4}\right)^{2}}{4 z_{6}^{2}} \geq 0,
$$

где $H_{A}, B_{A}, s_{A}$ - габаритные высота и ширина, толщина стенки торцевого сечения звена, к которому крепится корпус; $s_{B}$ - толщина стенки торцевого сечения конечного звена; $[\sigma]_{b r, k}$ - допусти-

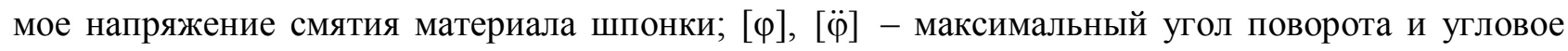
ускорение при разгоне конечного звена, регламентируемые техническим заданием на проектирование крана-манипулятора; $J_{\text {и }}$ - момент инерции вращающихся масс, приведенный к ротору соединения; $K_{\tau s}$ - коэффициент концентрации касательных напряжений при кручении шлицевого вала $[20] ;[\tau]_{t}, \sigma_{u s}$ - допустимое напряжение на срез и предел прочности материала ротора; $\psi-$ коэффициент учета неравномерности распределения нагрузки между шлицами и вдоль шлицев [5]; $m_{g}$ - модуль эвольвентного шлицевого соединения ротора с хомутом; $\sigma_{-1}-$ предел выносливости материала лопасти; $K$ - коэффициент снижения предела выносливости материала лопасти.

Результаты и их обсуждение. Предложенная математическая модель оптимального синтеза приводного шарнирного соединения была реализована применительно к конструкции трехзвенной шарнирно-сочлененной манипуляционной системы мобильной энергетической машины АСТ-4-А для сварки магистральных трубопроводов [7]. Ее внешний вид соответствует рис.1. Основные технические характеристики манипуляционной системы: номинальная грузоподъемность $7,5 \mathrm{\kappa H} ;$ крутящий момент $T_{t}=18 \mathrm{\kappa H} \cdot \mathrm{M} ;$ номинальное рабочее давление гидропривода $p_{1}=16$ МПа; длина рукояти 2,4 м; длина стрелы 3,6 м; максимальный угол поворота рукояти $50^{\circ}$.

На рис.4 приведены результаты оптимального синтеза приводного шарнирного соединения рукояти и стрелы крана-манипулятора в интервале крутящих моментов $T_{t}=3-22 \mathrm{\kappa H} \cdot \mathrm{м}$ при трех
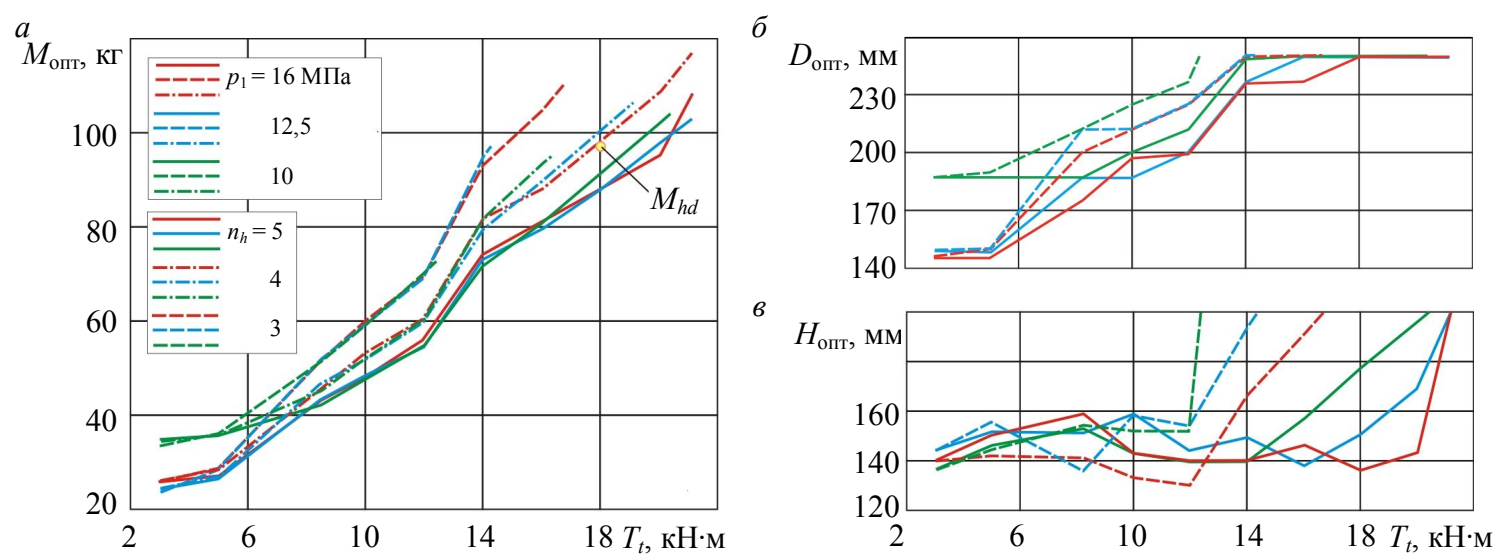

Рис.4. Оптимальные значения основных характеристик приводного шарнирного соединения: $a$ - масса; $\sigma$ - внутренний диаметр корпуса; в - длина корпуса 
стандартизованных уровнях рабочего давления гидросистемы $p_{1}=10 ; 12,5$ и 16 МПа. Более высокие значения $p_{1}$ не рассматривались, так как согласно данным $[4,6,22]$ при таких давлениях сложно обеспечить надежную герметизацию внутренних полостей устройства.

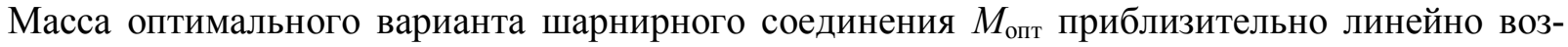
растает с ростом преодолеваемого крутящего момента $T_{t}$ (рис. $4, a$ ). При равном значении $T_{t}$ увеличение числа камер шарнирного соединения (увеличение числа лопастей $n_{h}$ ) позволяет ее снизить. Заметна следующая закономерность: рабочее давление $p_{1}$ оказывает весьма малое воздейст-

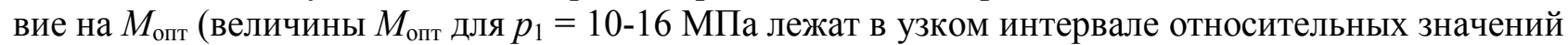
$\pm 3 \%$ ). Это обстоятельство имеет существенное практическое значение, так как позволяет использовать в гидросистеме крана-манипулятора меньшее рабочее давление и, соответственно, насосный агрегат меньшей мощности.

Применительно к рассматриваемому примеру возможен переход от штатного рабочего давления 16 МПа к пониженному рабочему давлению 12,5 или 10 МПа. Это заметно повышает энергоэффективность гидропривода, так как мощность установленного насосного агрегата может быть снижена приблизительно в 1,3 или 1,6 раз соответственно без ухудшения основных технических характеристик манипуляционной системы. Снижение рабочего давления гидросистемы также положительно сказывается на повышении коэффициента полезного действия гидропривода крана-манипулятора и снижении эксплуатационных затрат на его обслуживание. На рис.4, $a$ также указана масса силового гидроцилиндра, элементов его крепления и шарнирного соединения для штатной конструкции машины АСТ-4-А (точка $M_{h d}$ ), которая удаляется при переходе к использованию приводного шарнирного соединения. Видно, что использование десятикамерного соединения $\left(n_{h}=5\right)$ позволяет получить выигрыш по массе в пределах $\sim 9$ кг или $\sim 9 \%$. Использование восьмикамерного соединения $\left(n_{h}=4\right)$ приводит, наоборот, к увеличению массы манипуляционной системы, хотя весьма небольшому - в пределах 1-3 кг или 1-3 \%.

Оптимальные значения основных конструктивных размеров приводного шарнирного соединения $D_{\text {опт }}$ и $H_{\text {опт }}$ (рис. $4, \sigma, 6$ ) в зависимости от изменения крутящего момента $T_{t}$, рабочего давления $p_{1}$ и числа камер устройства $n_{h}$ изменяются достаточно разнопланово. Общая тенденция заключается в том, что с ростом $T_{t}$ эти размеры изменяются от минимальных до максимальных значений. В частности, внутренний диаметр корпуса изменяется от 146 до 250 мм, длина корпуса от 133 до 196 мм. Отмеченная особенность результатов оптимального синтеза обусловлена необходимостью встраивания приводного шарнирного соединения в спроектированную конструкцию манипуляционной системы, так как ее конструктивные размеры определяются приоритетно размерами поперечных сечений смежных звеньев, исходя из условий их прочности и жесткости.

Высота поперечного сечения сопрягаемых звеньев лимитирует предельные (наименьший и наибольший) значения диаметра корпуса, а ширина поперечного сечения - предельные значения длины корпуса приводного шарнирного соединения. Это подтверждается анализом расположения точек глобального минимума целевой функции $F(\{x\},\{z\})$ в допустимой области пространства управляемых параметров $\{x\}$, устанавливаемой системой принятых ограничений задачи оптимизации.

При малых значениях преодолеваемого крутящего момента $T_{t}<6-9$ кН·м точки глобального минимума лежат преимущественно на конструктивном ограничении (3), реже - на конструктивных ограничениях (3) и (6) или (3) и (4). При больших значениях $T_{t}$ они лежат на ограничении (7), т.е. оптимальный вариант устройства определяется усло-

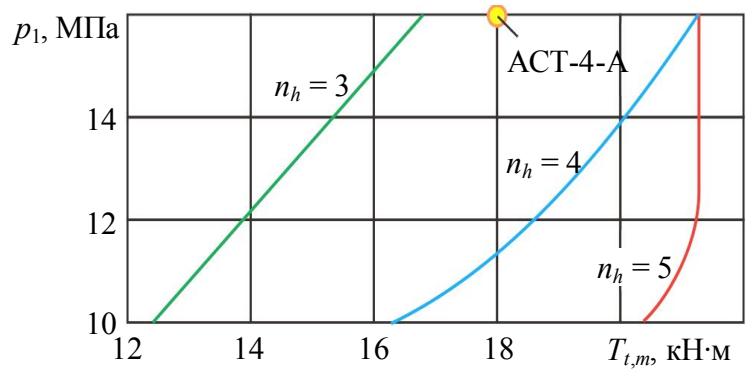

Рис.5. Предельный крутящий момент, развиваемый приводным шарнирным соединением вием обеспечения тягового усилия приводного шарнирного соединения. При предельных крутящих моментах $T_{t, m}$, развиваемых устройством, точки глобального минимума могут также лежать на конструктивном ограничении (5).

Значения предельных крутящих моментов $T_{t, m}$ в зависимости от преодолеваемого крутящего момента $T_{t}$ и рабочего давления гидросистемы $p_{1}$ приведены на рис.5. Здесь же указана точка, соответствующая характеристикам исходной для анализа машины ACT-4-А. Видно, что при замене штатной конструк- 
ции шарнирного соединения на приводное шарнирное соединение можно использовать десятикамерное устройство $\left(n_{h}=5\right)$ при любых значениях рабочего давления гидросистемы $p_{1}$ или восьмикамерное устройство $\left(n_{h}=4\right)$ при рабочем давлении $p_{1}=12,5$ или 16 МПа. Использование шестикамерного устройства $\left(n_{h}=3\right)$ невозможно, так как оно не обеспечивает необходимый крутящий момент $T_{t}=18 \mathrm{\kappa H} \cdot$ м (предельный крутящий момент при давлении 16 МПа составляет $\left.T_{t, m}=16,75 \mathrm{\kappa H} \cdot \mathrm{M}\right)$.

Выводы. При конструировании мобильных гидравлических кранов-манипуляторов рассмотренные приводные шарнирные соединения смежных звеньев шарнирно-сочлененных манипуляционных систем могут рассматриваться в качестве альтернативного варианта традиционных конструкций шарнирных соединений с выносными силовыми гидродвигателями.

При обеспечении равных грузо-высотных характеристик мобильного крана-манипулятора приводное шарнирное соединение позволяет исключить ряд эксплуатационных недостатков традиционного шарнирного соединения: развития с течением времени дополнительной динамической нагруженности металлоконструкции вследствие увеличения зазоров в соединении, снижения объема рабочей зоны крана из-за наличия выносных силовых гидродвигателей, появления отказов вследствие усталостного разрушения элементов узлов крепления силовых гидродвигателей к звеньям манипуляционной системы. При этом возможен перевод гидросистемы на меньшие уровни рабочего давления, что приводит к повышению энергоэффективности кранаманипулятора и коэффициента полезного действия гидропривода в целом, а также к снижению эксплуатационных затрат на обслуживание.

Благодарность. Исследование выполнено при поддержке Российского научного фонда (проект № 17-79-10274).

\section{ЛИТЕРАТУРА}

1. Александров М.П. Грузоподъемные машины. М.: МГТУ им. Н.Э. Баумана, 2000. 552 с.

2. Бандурин Р.А. Рынок кранов-манипуляторов в России // Проблемы современной экономики. 2015. № 26. С. 138-142.

3. Вайнсон А.А. Подъемно-транспортные машины строительной промышленности: Атлас конструкций. М.: Машиностроение, 1976. $152 \mathrm{c}$.

4. Галдин Н.С. Гидравлические схемы мобильных машин / Н.С.Галдин, И.А.Семенова. Омск: СибАДИ, 2013. 203 с.

5. Иосилевич Г.Б. Детали машин. М.: Машиностроение, 1988. 368 с.

6. Лагерев И.А. Проектирование поворотных гидродвигателей для манипуляционных систем мобильных машин на основе многокритериальной оптимизации / И.А.Лагерев, Е.А.Шатунова // Научно-технический вестник Брянского государственного университета. 2016. № 4. C. 34-51. DOI: 10.22281/2413-9920-2016-02-04-34-51

7. Лагерев И.А. Моделирование рабочих процессов манипуляционных систем мобильных многоцелевых транспортнотехнологических машин и комплексов / Брянский государственный университет. Брянск, 2016. 371 с.

8. Максаров В.В. Применение полимерных композитных материалов в узлах трения скважинных нефтяных насосов / В.В.Максаров, Ю.Ю.Ольт, В.А.Красный // Записки Горного института. 2015. Т. 211. С. 71-79.

9. Максаров В.В. Повышение точности изготовления силовых гидроцилиндров механизированных крепей на основе виброустойчивой инструментальной системы / В.В.Максаров, Ю.Ю.Ольт // Записки Горного института. 2015. Т. 214. С. 71-84.

10. Патент № 176269 РФ. Трехзвенный гидравлический кран-манипулятор / А.В.Лагерев, И.А.Лагерев. Опубл. 15.01.2018. Бюл. № 2 .

11. Серебрянский А.И. Силовой анализ работы шарнира манипулятора / А.И.Серебрянский, М.А.Мижевич // Актуальные направления научных исследований XXI века: теория и практика. 2014. T. 2. № 3. С. 238-241. DOI: 10.12737/4237

12. Повышение эксплуатационной надежности и расширение технологических возможностей перегрузочных машин морских портов / В.В.Суглобов, В.А.Михеев, И.А.Нефедов, П.В.Шиков // Известия Тульского государственного университета. Технические науки. 2009. № 2. С. 253-259.

13. Hydraulic drive systems / Non-linear control based on physical models. Lecture Notes in Control and Information Sciences. London: Springer, 2001. Vol. 260. P. 121-162. DOI: 10.1007/BFb0110344

14. Krasnyy V.A. Improving fretting resistance of heavily loaded friction machine parts using a modified polymer composition / V.A.Krasnyy, V.V.Maksarov, J.J.Olt // Agronomy Research. 2016. Vol. 14. № 1. P. 1023-1033.

15. Krasnyy V.A. Increase of wear and fretting resistance of mining machinery parts with regular roughness patterns / V.A.Krasnyy, V.V.Maksarov, J.J.Olt // Annals of DAAAM and Proceedings of the International DAAAM Symposium 27.2016. P. 151-156.

16. Lagerev A.V. Tool for Preliminary Dynamics and Stress Analysis of Articulating Cranes / A.V.Lagerev, I.A.Lagerev, A.A.Milto // International Rewiev on Modelling and Simulations. 2014. Vol. 7. № 4. P. 644-652. DOI:10.15866/iremos.v7i4.2045

17. Lagerev A.V. Preliminary Dynamics and Stress Analysis of Articulating Non-Telescoping Boom Cranes Using Finite Element Method / A.V.Lagerev, I.A.Lagerev, A.A.Milto // International Rewiev on Modelling and Simulations. 2015. Vol. 8. № 2. P. 223-227. DOI: 10.15866/iremos.v8i2.5713 
Оптимальный синтез приводных шарнирных соединений...

18. Maksarov V.V. Improving the accuracy of manufacturing of hydraulic power cylinders using vibration-proof cutting tool // Agronomy Research. 2015. Vol. 13. № 3. P. 671-679.

19. Peterson R.E. Stress concentration factors. N.Y.: John Wiley and Sons, 1974. 178 p.

20. Ravani B. Kinematics and Mechanisms. The Engineering Handbook. Boca Raton: CRC Press LLC, 2000. 324 p.

21. Shoham M. Hydraulic Drive Systems / A Textbook of Robotics 2: Structure, Control and Operation. Boston: Springer, 1984. P. 125-146. DOI: 10.1007/978-1-4615-9888-6 5

22. Singer D.B. The potential of structural mechanics research in crane boom design // SAE Technical Paper 600328, 1960. P. 9.

Авторы: А.В.Лагерев, д-р техн. наук, профессор, заместитель директора по научной работе НИИ фундаментальньхх и прикладных исследований, avl-bstu@yandex.ru (Брянский государственный университет имени академика И.Г.Петровского, Брянск, Россия), И.А.Лагерев, д-р техн. наук, доиент, проректор по инновачионной работе, lagerev-bgu@yandex.ru (Брянский государственный университет имени академика И.Г.Петровского, Брянск, Россия).

Статья поступила в редакиию 18.01.2018.

Статья принята к публикачии 28.06.2018. 